\title{
Importancia de la innovación y el emprendimiento en el ámbito académico ante el escenario de cambio tecnológico'
}

\section{Importance of innovation and entrepreneurship in the academic field in the face of the technological change scenario}

José Tulio Benavides-Peña²
Ana María Jaramillo-Quiceno

Recibido: 5-feb-2019

Aceptado: 18-sep-2019

\section{Resúmen}

El emprendimiento es una de las formas de transferencia del conocimiento desde la Universidad hacia la sociedad y esta fue la motivación para la presente investigación, la cual permitió reflexionar sobre la importancia del Emprendimiento de Base Tecnológica (Евт) como mecanismo de dicha transferencia. Esta reflexión permitió identificar cuáles son los elementos fundamentales para que la institución Unicatólica esté en capacidad de transferir el conocimiento que se genera, aprovechando su potencial en investigación, desarrollo tecnológico e innovación, con el fin de que se pueda así fortalecer su articulación con el entorno. La investigación fue de carácter exploratorio y descriptivo y para ello, se realizó consulta a fuentes secundarias y primarias, mediante entrevistas semiestructuradas aplicadas en Cali, Medellín, Bogotá y Barranquilla. La sistematización y análisis de la información evidenció los elementos centrales que serán insumo para la construcción de un modelo institucional para gestionar procesos de desarrollo tecnológico, innovación y emprendimiento, a partir de los procesos de formación y los resultados de investigación que se generan actualmente. Estos elementos son el desarrollo de capacidades institucionales desde el componente humano, físico y tecnológico, así como el monitoreo de tendencias y la articulación con el entorno mediante redes y plataformas nacionales e internacionales. Se logró finalmente concluir que el eje central para la articulación con el entorno está en lograr una coherencia estratégica y operativa entre el Proyecto Educativo Institucional (PEI), la investigación, el desarrollo tecnológico y la apropiación social del conocimiento.

Palabras clave: innovación, emprendimiento, cambio tecnológico, emprendimiento de base tecnológica, transferencia del conocimiento.

\section{Abstract}

Entrepreneurship is one of the forms of knowledge transfer from the University to society and this was what motivated this research, which allowed us to reflect on the importance of Technology Based Entrepreneurship (TBE), as a mechanism for transferring knowledge from the university to society. This reflection allowed to identify what are

1 Este artículo es uno de los resultados de investigación del proyecto "Factores que inciden en el desarrollo de la competencia tic por parte de los profesores de la Fundación Universitaria Católica Lumen Gentium de Cali", inscrito en la Licenciatura en Informática de unicatólica y al Grupo de investigación Educarte. Este proyecto se encuentra financiado por la Fundación Universitaria Católica Lumen Gentium- UnICATólica

2 Docente de la Fundación Universitaria Católica Lumen Gentium. Magíster en Gestión de Informática y Telecomunicaciones de la Pontificia Universidad Javeriana de Cali, Colombia. Correo electrónico: jbenavides@unicatolica.edu.co

3 Docente de la Fundación Universitaria Católica Lumen Gentium. Magíster en Gestión de Ciencia, Tecnología e Innovación de la Universidad de Antioquia, Colombia, Correo electrónico: amjaramillo@unicatolica.edu.co 
the fundamental elements for the Unicatólica institution to be able to transfer the knowledge that is generated, taking advantage of its potential in research, technological development and innovation, in order to strengthen its articulation with the environment. The research was exploratory and descriptive, and for this purpose, secondary and primary sources were consulted using semi-structured interviews applied in Cali, Medellín, Bogotá and Barranquilla. The systematization and analysis of the information evidenced the central elements that will act as input for the construction of an institutional model to manage technological development, innovation and entrepreneurship processes, based on training processes and research results generated. These elements are the development of institutional capacities from the human, physical and technological component, as well as monitoring trends and the articulation with the environment through national and international networks and platforms. It was finally concluded that the central axis for articulation with the environment is to achieve strategic and operational coherence between the Institutional Educational Project (PEI in Spanish), research, technological development and the social appropriation of knowledge.

Keywords: innovation, entrepreneurship, technological change, technology-based entrepreneurship, knowledge transfer.

\section{Introducción}

¿Cómo se imaginan el mundo en el 2050? La revista Smithsonian y el Pew Research Center realizaron una encuesta de opinión hace algunos años al respecto. Encontraron que el $71 \%$ de las personas piensa que el hombre encontrará una cura para el cáncer, 66 \% considera que las extremidades artificiales superarán las reales. Casi la mitad de los encuestados piensa que un humano será clonado en las próximas décadas y que la tecnología para leer los pensamientos de las personas existirá para el 2050 (Bustelo y Raygada, 2019).
Es este el escenario el presente y lo que vislumbran las perspectivas de futuro y a esto se abocan el desarrollo científico y tecnológico y la transferencia de conocimiento mediante la innovación y el emprendimiento, entre otros mecanismos.

Las universidades son laboratorios vivos, con capacidad de transformarse a sí mismas y a su entorno, pero no de forma aislada. Se demanda una apropiación tecnológica a nivel global para transferir el conocimiento con enfoque en el territorio.

Una amplia difusión de instrumentos estadísticos como las encuestas de innovación muestran que América Latina y el Caribe han visto un alto crecimiento en la producción de investigaciones y evidencia empírica que confirman la importancia de la innovación y el emprendimiento como motores del crecimiento (Aboal, Álvarez y Grazzi, 2019).

De acuerdo con lo anterior, se argumenta la necesidad de la Fundación Universitaria Católica Lumen Gentium- unicatólica de contar con un modelo que facilite el desarrollo de la innovación y el Emprendimiento de Base Tecnológica (Евт), con el fin de fortalecer sus capacidades y su articulación con el entorno. Para lograr lo anterior es preciso iniciar por un proceso de reflexión sobre los cambios del contexto global en la presente era.

Para esta investigación se realizó una exploración en fuentes secundarias que permitió sentar las bases conceptuales y así, en esta primera fase del estudio, diseñar un instrumento de consulta a fuentes primarias con el cual identificar los elementos fundamentales que se deben considerar y así generar las condiciones para que el ЕBт se convierta en un mecanismo de articulación con el entorno.

\section{Marco de referencia}

Se vive en una época de gran incertidumbre y riesgos cada vez mayores. El mundo actual es un ámbito en deconstrucción y reconstrucción, siempre 
inacabado. La revolución tecnológica y especialmente las llamadas Tecnologías de la Información y la Comunicación (TIC) han contribuido a romper las fronteras nacionales mediante redes virtuales que conectan diversos territorios, con frecuencia muy alejados entre sí (Zorro, 2015). Así, se presentan mayores retos para los territorios que aun estando interconectados y con las revoluciones industriales, no consiguen cerrar las brechas entre el centro y la periferia.

Pese al gran impulso que ha tenido ciencia, tecnología e innovación en el desarrollo socioeconómico mundial, nuestro planeta debate actualmente diferentes conflictos que necesariamente han aumentado la brecha social y económica que existe entre los estados miembros de la Organización para la Cooperación y el Desarrollo Económico (OCDE) y un resto de países que luchan por alcanzar un desarrollo humano adecuado. El desarrollo de una tecnología esencialmente para la guerra en el siglo $\mathrm{xx}$, sin considerar que en la mitad se jugaba el futuro de la humanidad, impuso -en algunos países- esquemas de investigación científica en los que se ha llegado a extremos tan riesgosos como el de poner en peligro la biosfera y con ello, la estabilidad física y biológica del planeta (Cypher, 1987; Hilleman, 2002; Fraser et al., 2001; Noah et al., 2002, citados en Domínguez y García, 2009, p. 9). De allí que sea fundamental lograr una consonancia entre el propósito del desarrollo científico y tecnológico y el desarrollo económico y social.

Adicionalmente, si bien los avances en algunas áreas de investigación científica han posibilitado un desarrollo progresivo de nuevas formas de comunicación, cognición y transferencia de información a interfaces inteligentes, las estructuras sociales y económicas del siglo xxI experimentan una crisis generada por la revolución en las Tecnologías de la Información y por modelos de desarrollo socioeconómico expansionista (Domínguez y García, 2009, p. 9). Con el paso del tiempo la transformación principal se fundamenta en el traslado de la producción basada en los bienes tangibles a los intangibles, el conocimiento se convierte en el eje de la ventaja competitiva. Sin embargo, no todas las economías logran los mismos niveles de desarrollo a la par y así se acrecientan las brechas cada vez más.

En las economías basadas en el conocimiento, en las que el factor básico de producción es el conocimiento, las universidades tienen el reto de potenciar la generación de innovaciones por medio de la investigación y dotar a la comunidad universitaria de las habilidades necesarias, básicamente a través de la docencia (Carlsson et al., 2002; Zaharia y Gibert, 2005; Van Looy, 2009; Perkmann y Walsh, 2007, citados en Guerrero y Urbano, 2017). En contraste, en las economías emprendedoras, en las que el emprendimiento es una de las principales palancas de producción, las universidades tienen el reto de desarrollar -simultáneamente- tanto el capital humano (intra) emprendedor como las iniciativas emprendedoras (Wright et al., 2007; Audretsch et al., 2008; 2009; Audretsch, 2009, citados en Guerrero y Urbano, 2017). Bien en uno u otro escenario, se observa un factor común que es el conocimiento como bien intangible y eje del desarrollo.

Se podría decir entonces que tradicionalmente, el común de las universidades evoluciona al atender a los cambios del entorno para adaptarse a estos. De otra parte, el dilema de la ética -que cada día se antepone más al discurso del cambio tecnológico y sus implicaciones en la sociedad- no puede ser menos importante y es necesario que cada actor busque orientar hacia una posición en la cual prime el interés de las mayorías por sobre el bien particular.

\section{Marco teórico}

\section{Esquemas de articulación de las universidades}

Los distintos actores sociales -entre ellos las universidades- hoy deben repensar sus modelos tradicionales para evolucionar hacia otros que sean más pertinentes a las nuevas demandas que nacen como resultado de los cambios del entorno global. En los últimos años se ha producido una transformación 
en la percepción social del papel de la Universidad como motor de desarrollo. Esto se debe a la adopción de modelos de transferencia basados en la valorización de la Investigación y Desarrollo, I+D universitarias y el fomento del espíritu emprendedor (Rubio de las Alas-Pumariño, 2014).

En este sentido, se reconoce ampliamente que las universidades son una pieza fundamental en los ecosistemas de emprendimiento e innovación. A pesar de ello, por su propia naturaleza, las universidades no destacan por una orientación demasiado emprendedora e innovadora. Por esta razón, a lo largo del tiempo las universidades se han enfrentado a importantes cambios estructurales y procesos de transformación en el desarrollo de sus principales actividades (docencia, investigación y transferencia de conocimiento a la sociedad), que requieren a su vez la participación activa de diversos actores (gobierno, emprendedores, inversores, entre otros) (Guerrero y Urbano, 2017).

Diversos estudios debaten el rol de la universidad entre la formación, investigación y proyección social, según sus intereses y enfoque particular. Sin embargo, en las últimas décadas, dadas las condiciones del contexto globalizado y la comprensión de la utilidad de la ciencia, tecnología e innovación como motores del desarrollo de los países, se está fortaleciendo el relacionamiento con el entorno, independientemente del enfoque. Así, se está incentivando cada vez más la articulación de la academia con el sector empresarial y gubernamental, este principio se conoce como la triple hélice en un primer momento (Etzkowitz y Leydesdorff, 1966, citados por Carayannis et al., 2012), el cual evolucionó con la perspectiva de varios autores alrededor del mundo y tornó en la hélice cuádruple que incorpora a la sociedad civil como un actor. Más recientemente, Carayannis et al. (2012) propusieron la quíntuple hélice, en la que se facilita el vínculo entre el conocimiento y la innovación para lograr un desarrollo sostenible, es decir, los autores propusieron como un quinto elemento las principales problemáticas mundiales para así dirigirse claramente hacia una investigación con propósito, con aplicación en el ámbito global.

Finalmente, Feldman y Kogler (2008, p. 446) consideran que debería haber una visión más amplia sobre las contribuciones de las universidades y no reducirlas a un "simple factor de producción", es decir, valorarlas como "fuerzas creativas de la economía”. De la misma manera, las universidades emprendedoras, representadas por la Universidad de Stanford o el Massachussets Institute of Technology (MIT), cumplen una serie de funciones en los sistemas regionales de innovación: crean spin-outs con alto valor tecnológico, realizan servicios de consultoría a las empresas locales, asesoran sobre políticas públicas, proveen al mercado laboral de personal altamente cualificado y formado, licencian nuevas tecnologías, atraen a investigadores, científicos y tecnólogos internacionales a la región, forman redes y estimulan la interacción social y el aprendizaje, desarrollan así la capacidad de innovación y apoyan el crecimiento económico regional (Salter y Martin, 2001; Lockett et al., 2005; Breznitz y Feldman, 2010, citados por Feldman y Kogler, 2008).

\section{Emprendimiento de base tecnológica e innovación}

Un estudio reciente realizado en los Estados Unidos reveló que la mayoría de las personas encuestadas piensa que el liderazgo innovador debe comenzar en las universidades de investigación. No obstante, ¿es en la academia donde actualmente nacen las ideas más transformadoras? Según datos arrojados en el Foro Económico Mundial (FEM) 2018, gracias a la investigación realizada por Times Higher Education, 27 de las universidades más prestigiosas e innovadoras del mundo generan más investigación que casi todos los países del mundo (excepto Estados Unidos y China). Sin embargo, no es suficiente, es necesario que más instituciones se sumen al esfuerzo (Guijosa, 2018). 
Asimismo, es cierto que las universidades, que a su vez forman parte de sus respectivos ecosistemas de emprendimiento e innovación, requieren de una actitud proactiva y de una interconexión constante con sus stakeholders (Guerrero y Urbano, 2017), lo que implica contar con esquemas de gobernanza claros.

Esto facilita el desarrollo del emprendimiento, el cual es cada vez más un mecanismo de transferencia de conocimiento para las universidades. El objetivo es que el alumnado que salga de sus aulas sea capaz de emprender, y no solo en el sentido de crear una empresa, sino que tenga la capacidad de innovar en todas aquellas tareas que su desempeño profesional requiera (Segarra Ciprés et al., 2017). De allí la importancia de la alineación con los principios institucionales y la incorporación de prácticas, tanto en el currículo como fuera de él, para promover la innovación y el emprendimiento. Necesitamos que la universidad genere emprendedores e innovadores.

"Por otro lado, la colaboración, base de esta nueva aventura, está llamada a ser una de las claves del éxito en el futuro"‘ (Huete, 2018). Y esta es una necesidad a nivel global, pues en la era de la economía digital son muchas las capacidades que se requiere desarrollar en los estudiantes y también en los docentes, lo que trasciende el currículo de formación tradicional de las universidades.

En Colombia, aunque para el gobierno Nacional los emprendedores contribuyen al crecimiento a través de la creación de empleo y el desarrollo de nuevos productos, hay barreras para acceder al financiamiento y conseguir oportunidades de crecimiento, además, muchas de las fuentes de financiación son informales (Departamento Nacional de Planeación [DNP], 2019). Esto implica un importante reto para la universidad que anida en los procesos de mentoría y etapas tempranas como la ideación,

4 Las declaraciones provienen de una entrevista del 17 de enero del 2018 realizada por el portal de innovación Innovaspain al Doctor Álvaro Bernad, Director de Innovación Disruptiva de Calidad Pascual, en el marco de la implementación de Madrid Innovation Driven Ecosystem (MIDE), una plataforma colaborativa y sin ánimo de lucro cuya misión es acelerar la economía, fomentar la innovación y el emprendimiento, y crear empleo en la capital española. hasta la formalización y el acompañamiento para la consolidación de empresas que sean sostenibles en el tiempo.

Se denominan Empresas de Base Tecnológica aquellas que basan su actividad en las aplicaciones de nuevos descubrimientos científicos o tecnológicos para la generación de nuevos productos, procesos o servicios. La importancia de estas empresas para potenciar el tejido tecnológico y el desarrollo económico, favorecer la creación de empleo de alta cualificación, aportando un alto valor añadido al entorno industrial, ha hecho que las universidades y otras instituciones públicas de investigación les dediquen una creciente atención como auténticos motores en la transferencia de conocimiento. (Díaz et al., 2007, p. 132)

Unas condiciones favorables para que se refuerce la transferencia de conocimiento y se establezcan mecanismos de cooperación entre la Universidad y el sector productivo inciden directamente en las posibilidades de crear Евт y de entablar las relaciones entre estas empresas y la institución de origen [...] Crear una empresa generalmente significa dar valor a una oportunidad que hubiese quedado en las estanterías. Significa también hacer partícipes a profesores, ex alumnos y $\mathrm{PhD}$ de un proyecto empresarial, mientras se mantiene la relación con la [u] niversidad y se contratan investigaciones con el departamento de origen. Lo anterior, permite permeabilizar los grupos de investigación con el tejido productivo. Pero, fundamentalmente, crear una empresa ofrece una nueva vía para el desarrollo profesional del personal universitario, constituyendo un nuevo reto profesional y personal que les permitirá: desarrollar su vocación emprendedora, valorizar los resultados de su trabajo de investigación, aplicar sus desarrollos científicos en el mercado y contribuir al desarrollo de su entorno social. (MEC, 2008)

El punto de partida para la construcción de la noción de medio innovador es la constatación de que, no solo la actividad económica en general, sino específicamente los procesos de innovación tecnológica y por ende, las empresas que los generan se tienden a concentrar en ciertos territorios, sobre todo de carácter urbano-metropolitano. En especial, en estos espacios se dan las precondiciones que propician el surgimiento de innovaciones: acumulación de know-how técnico, recursos humanos calificados, infraestructuras tecnológicas, universidades y centros de apoyo, capital de riesgo, etc., además de las ventajas que proporciona la proximidad espacial de las firmas innovadoras (Moncayo, 2002, p. 64). 


\section{Metodología}

Se ha de aclarar que el análisis del presente artículo se basa en los logros y aprendizajes de la fase exploratoria de una investigación en curso, que comprende en total cuatro (4) fases: exploratoria, descriptiva, diseño e implantación.

La metodología de la fase exploratoria consistió en primer lugar, en la consulta de fuentes secundarias, lo que permitió identificar conceptos para tener un contexto claro de las temáticas centrales de la investigación en el ámbito global. Con esta información, se priorizaron las temáticas coherentes con la filosofía y direccionamiento institucional y se diseñaron instrumentos de consulta a actores estratégicos de las ciudades principales del país: Bogotá, Medellín, Cali y Barranquilla. Esto por ser las que cuentan con un mayor desarrollo en sus ecosistemas de Investigación, Desarrollo Tecnológico e innovación (I+D+i).

La consulta a fuentes primarias incluyó actores del gobierno e instituciones, empresarios y directivos. Para seleccionar a los empresarios se utilizó la lista de "Las 100+ de las iniciativas clúster" (Cámara de Comercio de Cali, 2018), se tomó en cuenta un $30 \%$ del total, con el fin de hacer una muestra significativa que cubriera más de la cuarta parte de la población seleccionada. Con respecto a actores de gobierno e instituciones se seleccionaron los más representativos en los ecosistemas de $\mathrm{I}+\mathrm{D}+\mathrm{i}$ al nivel nacional, en relación con las temáticas centrales identificadas, a juicio de los investigadores.
A continuación, se exponen los resultados de la aplicación de esta metodología y el análisis que pretende motivar la reflexión aquí planteada.

\section{Resultados}

Como se enunció, los principales resultados de esta reflexión han sido los aportes obtenidos de cada uno de los actores interesados, lo cual le da un enfoque claro al diseño del modelo para contextualizar los intereses, necesidades y proyección estratégica de la institución y sus aliados.

\section{Consulta de actores externos}

Se presenta aquí la síntesis de los resultados de la entrevista semiestructurada aplicada a los actores gubernamentales, instituciones y empresas.

Como se muestra en la tabla 1, esa fue la población objeto de investigación; sin embargo, al realizar la indagación de campo, se llevó a cabo una convocatoria a participar a todas las empresas descritas en la muestra, con el objetivo de obtener respuesta de al menos un $30 \%$ del total. Adicionalmente se optó por realizar la aplicación del instrumento a empresas que estuvieran entre las "100+" de los clústers y también a las empresas que fueran multinacionales con opción de mercado dentro de los clústers, con el fin de hacer que la información fuera equiparable.

Tabla 1. Las 100+ de las seis iniciativas clúster divididas por clústers

\begin{tabular}{llllll}
$\begin{array}{l}\text { Belleza y cuidado } \\
\text { personal }\end{array}$ & \multicolumn{1}{c}{ Bioenergía } & \multicolumn{1}{c}{$\begin{array}{c}\text { Excelencia } \\
\text { clínica }\end{array}$} & \multicolumn{1}{c}{ Sistema moda } & Proteína blanca & Macrosnacks \\
3 Tecnoquímicas & 2 EPSA - & 18 Coomeva & 19 Stf Group & 16 Pollos el & 1 Colombina \\
S.A. & $\begin{array}{l}\text { empresa de } \\
\text { energía del } \\
\text { pacífico S.A. E.S.P. }\end{array}$ & $\begin{array}{l}\text { Medicina } \\
\text { Prepagada S.A. }\end{array}$ & S.A. & Bucanero S.A. & S.A. \\
& & & &
\end{tabular}




\begin{tabular}{|c|c|c|c|c|c|}
\hline $\begin{array}{c}\text { Belleza y cuidado } \\
\text { personal }\end{array}$ & Bioenergía & $\begin{array}{l}\text { Excelencia } \\
\text { clínica }\end{array}$ & Sistema moda & Proteína blanca & Macrosnacks \\
\hline $\begin{array}{l}4 \text { Colgate } \\
\text { palmolive } \\
\text { compañía }\end{array}$ & $\begin{array}{l}5 \text { Gases de } \\
\text { Occidente S.A. } \\
\text { E.S.P. }\end{array}$ & $\begin{array}{l}20 \text { Fundación } \\
\text { Valle Del Lili }\end{array}$ & 41 Sí S.A.S. & $\begin{array}{l}21 \text { Mac Pollo } \\
\text { - Avidesa De } \\
\text { Occidente S.A. }\end{array}$ & $\begin{array}{l}11 \text { Harinera } \\
\text { del Valle S.A. }\end{array}$ \\
\hline $\begin{array}{l}7 \text { Johnson \& } \\
\text { Johnson de } \\
\text { Colombia S.A. }\end{array}$ & 6 Emcali & $\begin{array}{l}25 \text { Centro } \\
\text { Médico } \\
\text { Imbanaco de } \\
\text { Cali S.A. }\end{array}$ & $\begin{array}{l}46 \text { Calzatodo } \\
\text { S.A. }\end{array}$ & $\begin{array}{l}23 \text { Incubadora } \\
\text { Santander S.A. }\end{array}$ & $\begin{array}{l}30 \text { Productos } \\
\text { Yupi S.A.S. }\end{array}$ \\
\hline $\begin{array}{l}10 \text { Cartón de } \\
\text { Colombia S.A. }\end{array}$ & $\begin{array}{l}8 \text { Ingenio del } \\
\text { Cauca S.A.S. }\end{array}$ & 27 Comfandi & 47 Quest S.A.S. & $\begin{array}{l}33 \text { Cervalle - } \\
\text { Cerdos Del Valle } \\
\text { S.A. }\end{array}$ & $\begin{array}{l}37 \text { Comes- } \\
\text { tibles Aldor } \\
\text { S.A.S. }\end{array}$ \\
\hline $\begin{array}{l}14 \text { Ingredión } \\
\text { Colombia S.A. }\end{array}$ & $\begin{array}{l}9 \text { Riopaila } \\
\text { Castilla S.A. }\end{array}$ & $\begin{array}{l}29 \text { Dumian } \\
\text { Medical S.A.S. }\end{array}$ & $\begin{array}{l}48 \text { Venus } \\
\text { Colombiana } \\
\text { S.A. }\end{array}$ & $\begin{array}{l}35 \text { Santa Anita } \\
\text { Nápoles S.A. }\end{array}$ & $\begin{array}{l}50 \text { Impor- } \\
\text { taciones y } \\
\text { Exportaciones } \\
\text { Fénix S.A.S. }\end{array}$ \\
\hline 26 Sucroal S.A. & 12 Manuelita S.A. & $\begin{array}{l}31 \text { Sinergia } \\
\text { Global En } \\
\text { Salud S.A.S. }\end{array}$ & $\begin{array}{l}63 \text { Eka } \\
\text { Corporación } \\
\text { S.A. }\end{array}$ & $\begin{array}{l}40 \text { Nutriavíco- } \\
\text { la - Nutrientes } \\
\text { Avícolas S.A. }\end{array}$ & $\begin{array}{l}51 \text { Confitecol } \\
\text { S.A. }\end{array}$ \\
\hline $\begin{array}{l}28 \text { Carvajal } \\
\text { Empaques S.A. }\end{array}$ & $\begin{array}{l}13 \text { Ingenio } \\
\text { Providencia S.A. }\end{array}$ & $\begin{array}{l}38 \text { Clínica De } \\
\text { Occidente S.A. }\end{array}$ & $\begin{array}{l}70 \text { Spataro } \\
\text { Nápoli S.A. }\end{array}$ & $\begin{array}{l}43 \text { Carval - } \\
\text { Vallecilla B. y } \\
\text { Vallecilla M. y } \\
\text { Cía. S.C.A. }\end{array}$ & $\begin{array}{l}59 \text { Fabrifolder } \\
\text { S.A.S. }\end{array}$ \\
\hline $\begin{array}{l}34 \text { Reckitt } \\
\text { Benckiser } \\
\text { Colombia S.A. }\end{array}$ & $\begin{array}{l}15 \text { Centelsa - } \\
\text { Cables de Energía } \\
\text { y de Telecomuni- } \\
\text { caciones S.A. }\end{array}$ & $\begin{array}{l}52 \text { Clínica } \\
\text { Farallones S.A. }\end{array}$ & $\begin{array}{l}73 \text { Fábrica } \\
\text { de Calzado } \\
\text { Rómulo Ltda. }\end{array}$ & $\begin{array}{l}45 \text { Super pollos } \\
\text { del Galpón S.A.S. }\end{array}$ & $\begin{array}{l}69 \text { Manitoba } \\
\text { Ltda. }\end{array}$ \\
\hline 39 Recamier S.A. & $\begin{array}{l}17 \text { Carvajal Pulpa } \\
\text { Y Papel S.A. }\end{array}$ & $\begin{array}{l}53 \text { Angiografía } \\
\text { de Occidente } \\
\text { S.A. }\end{array}$ & $\begin{array}{l}80 \text { C.I. Manu- } \\
\text { facturas Model } \\
\text { Internacional } \\
\text { S.A.S. }\end{array}$ & $\begin{array}{l}56 \text { Avícola Santa } \\
\text { Rita S.A.S. }\end{array}$ & $\begin{array}{l}71 \text { Agrícola } \\
\text { Himalaya S.A. }\end{array}$ \\
\hline $\begin{array}{l}42 \text { Belleza } \\
\text { Express S.A. }\end{array}$ & & $\begin{array}{l}55 \text { Hospital en } \\
\text { Casa S.A. }\end{array}$ & $\begin{array}{l}84 \text { Textiles } \\
\text { Acrilán Ltda. }\end{array}$ & $\begin{array}{l}57 \text { Productora } \\
\text { Nacional Avícola } \\
\text { S.A. }\end{array}$ & $\begin{array}{l}74 \text { Bengala } \\
\text { Agrícola } \\
\text { S.A.S. }\end{array}$ \\
\hline $\begin{array}{l}44 \text { Plásticos } \\
\text { Especiales S.A.S. }\end{array}$ & 22 Mayagüez S.A. & $\begin{array}{l}61 \text { Hemato } \\
\text { Oncólogos } \\
\text { S.A. }\end{array}$ & $\begin{array}{l}85 \text { Calzado } \\
\text { Simeon - } \\
\text { Manufacturas } \\
\text { Af S.A.S. }\end{array}$ & $\begin{array}{l}58 \text { Industria de } \\
\text { Alimentos Carbel } \\
\text { S.A. }\end{array}$ & $\begin{array}{l}75 \text { Tecnoplast } \\
\text { S.A.S. }\end{array}$ \\
\hline $\begin{array}{l}54 \text { MM Packaging } \\
\text { Colombia S.A.S. }\end{array}$ & $\begin{array}{l}24 \text { Ingenio la } \\
\text { Cabaña S.A. }\end{array}$ & $\begin{array}{l}65 \text { Clínica } \\
\text { Palma Real } \\
\text { S.A.S }\end{array}$ & $\begin{array}{l}86 \text { Aritex de } \\
\text { Colombia S.A.S. }\end{array}$ & $\begin{array}{l}60 \text { Fogel Andina } \\
\text { S.A.S. }\end{array}$ & $\begin{array}{l}78 \text { Productos } \\
\text { Alimenticios } \\
\text { la Locura S.A. }\end{array}$ \\
\hline
\end{tabular}


(Viene de la página 121)

\begin{tabular}{|c|c|c|c|c|}
\hline $\begin{array}{c}\text { Belleza y cuidado } \\
\text { personal }\end{array}$ & Bioenergía & $\begin{array}{l}\text { Excelencia } \\
\text { clínica }\end{array}$ & Sistema moda & Proteína blanca Macrosnacks \\
\hline 64 Aptar Cali S.A.S. & $\begin{array}{l}32 \text { Ingenio } \\
\text { Pichichí S.A. }\end{array}$ & $\begin{array}{l}67 \text { Gilmédica } \\
\text { S.A. }\end{array}$ & $\begin{array}{l}87 \text { Calzado } \\
\text { Reindeer - } \\
\text { Comercializadora } \\
\text { Aviv S.A.S. }\end{array}$ & $\begin{array}{l}62 \text { Carnes Frías } \\
\text { Enriko Ltda. }\end{array}$ \\
\hline $\begin{array}{l}68 \text { Plafilms } \\
\text { Internacional - } \\
\text { Plastic Films } \\
\text { Internacional S.A. }\end{array}$ & 36 Vatia S.A. E.S.P. & $\begin{array}{l}77 \text { Dime - } \\
\text { Clínica Neuro- } \\
\text { cardiovascular } \\
\text { S.A. }\end{array}$ & 90 Evacol S.A.S. & $\begin{array}{l}66 \text { Alimentos } \\
\text { La Cali S.A. }\end{array}$ \\
\hline $\begin{array}{l}72 \text { Agraf Industrial } \\
\text { S.A. }\end{array}$ & $\begin{array}{l}49 \text { Imecol - } \\
\text { Ingeniería } \\
\text { Maquinaria y } \\
\text { Equipos de } \\
\text { Colombia S.A. }\end{array}$ & $\begin{array}{l}88 \text { Red de } \\
\text { Salud del } \\
\text { Norte } \\
\text { Empresa Social } \\
\text { del Estado }\end{array}$ & $\begin{array}{l}93 \text { Confeccio- } \\
\text { nes Salomé } \\
\text { Ltda. }\end{array}$ & $\begin{array}{l}81 \text { Industrias } \\
\text { Refridcol S.A. }\end{array}$ \\
\hline $\begin{array}{l}82 \text { Laboratorios OSA } \\
\text { S.A.S. }\end{array}$ & $\begin{array}{l}76 \text { Dicel - } \\
\text { Distribuidora y } \\
\text { Comercializadora } \\
\text { de Energía } \\
\text { Eléctrica S.A. E.S.P. }\end{array}$ & $\begin{array}{l}89 \text { Amanecer } \\
\text { Médico S.A.S. }\end{array}$ & $\begin{array}{l}95 \text { Denim } \\
\text { Factory S.A. }\end{array}$ & \\
\hline $\begin{array}{l}83 \text { Droguería } \\
\text { San Jorge ltda. }\end{array}$ & $\begin{array}{l}79 \text { Riopaila energia } \\
\text { S.A.S. E.S.P }\end{array}$ & $\begin{array}{l}98 \text { Enertotal } \\
\text { S.A. E.S.P. }\end{array}$ & $\begin{array}{l}97 \text { Femme } \\
\text { International } \\
\text { S.A.S. }\end{array}$ & \\
\hline $\begin{array}{l}91 \text { Indugráficas } \\
\text { S.A.S. }\end{array}$ & $\begin{array}{l}99 \text { Clínica de Oftal- } \\
\text { mología de Cali S.A. }\end{array}$ & & & \\
\hline $\begin{array}{l}92 \text { Fragancias y } \\
\text { Sabores S.A. }\end{array}$ & $\begin{array}{l}100 \text { Enerco S.A. } \\
\text { E.S.P. }\end{array}$ & & & \\
\hline $\begin{array}{l}94 \text { Sumiquim - } \\
\text { Suministros } \\
\text { Quimicos Ltda. }\end{array}$ & & & & \\
\hline
\end{tabular}

96 Imágenes

Gráficas S.A

Fuente: elaboración propia (2018)

Al realizar la invitación, no todas las empresas aceptaron participar, por lo que se involucraron algunas que no aparecen en el listado. Las empresas entrevistadas fueron: Comfandi, Río Paila Castilla, Coomeva, sgs Colombia S.A.S, Tecnoquímicas S.A, Unilever, Carvajal de Colombia, Abbott -Lafrancol S.A.S, Río Paila Agrícola, Fundación Valle del Lili, Johnson \& Johnson, Almacenes Sí, Grupo Mayorista S.A.- Herpo, Maquite S.A., Crepes \& Waffles, Clínica RIE,Clínica Becerra y Soto, Decko Deluxe, Franquicia de inversiones tiendas KOAJ.
Las instituciones y entidades de gobierno fueron: Universidad del Valle (2 entrevistas), Centro Internacional de Agricultura Tropical (CIAT), Sena sede Cartago, sena sede Cali, (2 entrevistas), Gobernación del Valle, Secretaría de Tic, Emcali, Puj Campus Nova, Universidad de San Buenaventura de Cali, Instituto de Emprendimiento Sostenible de la Universidad ean, Ministerio de Tecnologías de la Información y las Comunicaciones de Colombia (Mintic) (2 entrevistas) y Universidad Simón Bolívar (Macondo Lab). 
En total se realizaron 32 entrevistas a actores externos de las ciudades Cali, Bogotá y Barranquilla. Cali fue la ciudad central en la cual se aplicó el mayor número de entrevistas. En Bogotá y Barranquilla se realizó una referenciación con actores estratégicamente seleccionados de los respectivos ecosistemas de Ciencia, Tecnología e Innovación (CteI).

La interpretación de la información recopilada se generó a través de la experiencia en campo y el soporte del software Atlas ti 8, como herramienta de análisis cualitativo idóneo para este tipo de investigaciones, dado que en su mayoría, se formularon preguntas abiertas. Se muestra el word cloud (nube de palabras) resultado de este procesamiento con el software en la figura 1:

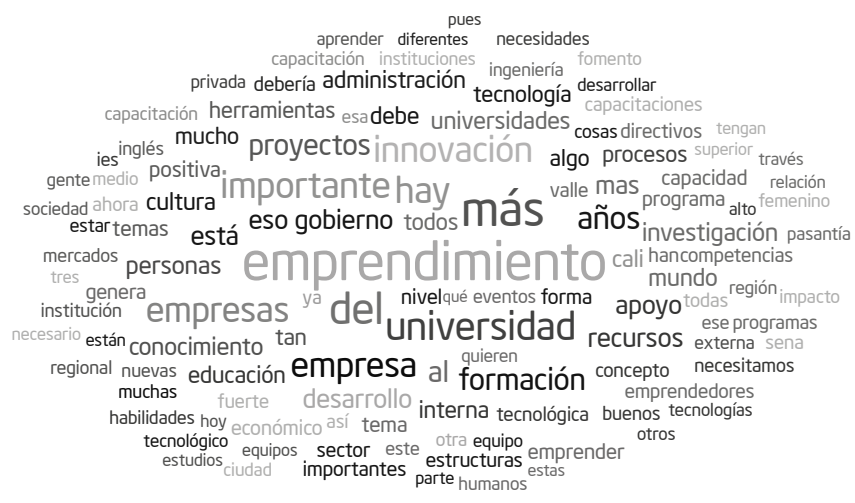

Figura 1. Word cloud de las entrevistas semiestructuradas a actores externos

Fuente: elaboración propia a partir de los datos de fuentes primarias (2018)

Durante la sistematización de las entrevistas se sugirió la necesidad de conocer cuál es la temática o concepto que a juicio de los entrevistados cobraría más relevancia y mediante la herramienta Word Cloud se determinó que "emprendimiento" está en primer lugar con 119 repeticiones, le siguen: "universidad" con 80; "innovación" con 70; "gobierno" con 40; "empresa" con 64; "desarrollo" con 39; "investigación" con 35; y "tecnología" con 32 apariciones en la transcripción de las entrevistas.

A continuación, se destacan los testimonios más representativos entre los recopilados:

\section{¿Cómo se ve el papel del gobierno en la región?}

Dentro del contexto de análisis generado desde el aplicativo, el gobierno es visto como el ente responsable de ejercer autoridad y apoyo económico:

El gobierno tiene una responsabilidad en cuanto a la asignación de recursos para que las personas puedan tener una educación universitaria y al final eso va a representar un impacto en la sociedad, en lo económico y en todo lo que tiene que ver, porque al final eso dinamiza la economía. (transcripción de entrevista, 2018)

El gobierno debe prestarnos los medios para que podamos desarrollar actividades o proyectos para la sociedad y también que en la empresa podamos ejercer lo que estudiamos y podamos crecer y aportar mucho a la empresa. (transcripción de entrevista, 2018)

El gobierno debería dar un aporte significativo para apoyar a que la educación y el desarrollo de las empresas de la región sigan mejorando y progresando, con lo cual a su vez aporta a la sociedad civil, en general para tener una mejoría en su calidad y condición de vida. (transcripción de entrevista, 2018)

\section{¿Cómo se ve el papel de la universidad en la región?}

Hemos encontrado que el relacionamiento universidad-Estado-empresa debe estar en las tres áreas, desde poder generar investigación pertinente para la definición de política pública e investigación en áreas soportadas en necesidades reales del entorno, hasta vincular en procesos de educación a las empresas a través de esquemas de formación, a partir de retos. (transcripción de entrevista, 2018)

Debe existir un trabajo transectorial, de manera que desde la academia se generen estrategias para aumentar el desarrollo, la productividad y el desempeño dentro de las organizaciones y las empresas. (transcripción de entrevista, 2018)

La mirada del sector académico es muy distinta a la del sector productivo y a la del sector estatal, entonces, suelen buscar objetivos que distan mucho el uno del otro, en vez de lograr combinar sus miradas, que es la intención teórica de la triple hélice. (transcripción de entrevista, 2018) 
Las universidades nos preparan muchas veces para salir a la industria o para continuar investigando, pero debido a las limitaciones económicas muchas personas no lo pueden hacer y cuando entran a una empresa se dan cuenta que mucho de su conocimiento adquirido no lo van a aplicar, entonces se limitan mucho a las funciones que tienen que cumplir dentro de la compañía y no hay ese apoyo. (transcripción de entrevista, 2018)

Es importante que se trabaje en un cambio de mentalidad. Las universidades hoy en día presionan a los estudiantes a ser emprendedores y las empresas también necesitan empleados, el sector está demandando desarrolladores y no los hay, los jóvenes quieren salir a la gerencia y no a desarrollar, que es lo que permite que se consoliden como profesionales y puedan luego crecer y tener sus propios negocios. (transcripción de entrevista, 2018)

\section{¿Quién debe promover el emprendimiento?}

El tema es determinante con diferentes roles o acciones, pero todos, los gobiernos local y regional, deben enfocarse en facilitar y generar condiciones para el desarrollo del emprendimiento. (transcripción de entrevista, 2018)

La academia tiene una etapa fundamental sobre la sensibilización, el fomento y el acompañamiento de las etapas más tempranas dentro del proceso emprendedor y otros actores que se han venido desarrollando. (transcripción de entrevista, 2018)

Lo vital es cómo articulamos la oferta de ese ecosistema, cómo entendemos el rol de cada uno y las grandes empresas. Las empresas existentes también tienen un rol muy importante dentro de la generación de espacios y esquemas que fomenten y apoyen el desarrollo de nuevos empresarios, tanto en políticas de compra, como en la articulación de sus cadenas de valor, esquemas de apoyo y fomento. (transcripción de entrevista, 2018)

\section{¿Qué ventaja competitiva identifica en el Valle del Cauca en comparación con los ecosistemas de otras regiones?}

En este análisis se destacaron los aspectos positivos y otros por mejorar, como se muestra en la tabla 2 a continuación:
Tabla 2. Aspectos positivos y por mejorar del ecosistema de emprendimiento del Valle del Cauca

\section{Aspectos positivos}

Aspectos por mejorar

- Por ser Cali la ciudad más - Falta confianza en los grande del suroccidente actores y formación. La colombiano tiene una alta región no está bien artidensidad de universidades, culada y no se conecta inclusive centros de investi- a los emprendedores gación que no se encuentran con fondos de capital en otras regiones como Nari- de inversión de la forño, Cauca o Putumayo, que ma en que lo requieren. realmente no cuentan a ve- - En la región estamos ces con vías de acceso o con buenos aeropuertos.

- El puerto de Buenaventura, que de una u otra forma debe ser un factor diferencial para la región.

- Cali tiene ventajas por mano de obra calificada y entidades comprometidas. El gobierno está trabajando y jalonando para que esto se dé. Hay un pacto de emprendimiento. Además hay ciertos fondos de las universidades.

- La ubicación y el relacionamiento estratégico con relación al Pacífico y la frontera sur, gran riqueza cultural e interdisciplinaria, ya que Cali es un receptor de todo esto.

- Somos una industria azucarera y en gran medida tenemos caña, tenemos una industria dulcera, confitera muy grande y también un sector de servicios muy fuerte y de licores.

- Tiene una ventaja competitiva frente a emprendimientos relacionados con sectores $\mathrm{O}$ áreas en las que el Valle del Cauca es fuerte a nivel de ingenios y de industria alimentaria que hay en Cali y en el Valle del Cauca en general. muy débiles en áreas de Ciencia, Tecnología, Ingeniería y Matemática (STEM) y eso hace que no se genere investigación de otro nivel.

- Se percibe poco interés del gobierno local en fortalecer e incentivar el emprendimiento. - Toca madurar un poco más el ecosistema. Aún pensamos solo en el beneficio económico y puede ser por las mismas necesidades de la región. Es una vía para conseguir recursos de subsistencia, no una realización personal y profesional. - Veo crecimiento en Cali con respecto al emprendimiento en los últimos años, pero frente a otras ciudades como Medellín estamos rezagados, hemos adoptado buenas prácticas de otras ciudades más desarrolladas en ese tema y que se vienen implementando en Cali.

Fuente: elaboración propia a partir de las entrevistas (2018) 


\section{¿Cómo se promueven el emprendimiento y la innovación?}

Se logró identificar al interior de las empresas algunos procesos encaminados a la innovación y el emprendimiento:

- Comfandi: cuenta con una célula de innovación.

- Los ingenios azucareros: a través de los procesos de investigación que contratan de Cenicaña.

- Tecnoquímicas: fomentan la incorporación de plataformas digitales que facilitan procesos.

- Coomeva: como grupo empresarial está trabajando en un caso de negocio, indagan sobre lo que hoy tienen para empresarios y la gente que apenas está iniciando; para ver si realmente se cumple con las expectativas o qué deben fortalecer a nivel de producto, servicios y canales de acceso.

- Abbott - Lafrancol S.A.S: tiene una planta que simula el campo industrial para generar prototipos de fabricación y sacar adelante un producto, para poder entregar un producto estandarizado con la mejor ruta, que sea seguro.

- En el resto de empresas: destacan la educación externa, capacitaciones internas, eventos de emprendimiento y apoyo económico.

Las estrategias del gobierno identificadas fueron:

El Ministerio de Tecnologías de la Información y las Comunicaciones (MinTIC) trabajan en cuatro (4) líneas:

1. Línea de financiamiento para el desarrollo de contenidos. Se hace a través de convocatorias, las más representativas son:
- Crea Digital: financia contenidos educativos y culturales;

- Animación, videojuegos y transmeda;

- Niumedia, en cooperación con el canal Media font para producir contenidos entre ambos países.

2. Línea de formación y generación de capacidades a través de laboratorios. Son en total 37 laboratorios alrededor de todo el país, dos de ellos en Cali (sena y Parquesoft).

3. Internacionalización, se lleva a las empresas a los principales eventos de animación y videojuegos y programa de formación para desarrollar las habilidades exportadoras.

4. Línea de difusión con su principal evento Colombia 4.0.

Adicionalmente, este Ministerio cuenta con una línea de formación y generación de capacidades a través de laboratorios. Son en total 37 laboratorios alrededor de todo el país, dos de ellos en Cali (sENa y Parquesoft).

- Valle inN: desde la Gobernación del Valle del Cauca acompaña todos los procesos con metodología de emprendimiento, y la Secretaría de Tic brinda asesoría técnica para que los emprendedores sepan si sus ideas son o no viables, en particular para el sector TIC.

\section{¿Qué competencias debe tener un emprendedor?}

El análisis de esta categoría se hace desde el paradigma del emprendimiento realizado por Rodríguez (2007) en el artículo "El paradigma de las competencias hacia la educación superior". 
Tabla 3. Competencias que debería tener el emprendedor

\begin{tabular}{|c|c|c|c|}
\hline Desarrollo de actitudes & $\begin{array}{c}\text { Conocimientos } \\
\text { generales y específicos } \\
\text { (saberes) }\end{array}$ & $\begin{array}{l}\text { Destrezas técnicas y } \\
\text { procedimentales }\end{array}$ & Competencias sociales \\
\hline Ser & Saber & Saber hacer & Convivir \\
\hline $\begin{array}{l}\text { - Responsabilidad } \\
\text { - } \text { Perseverancia } \\
\text { - } \text { Empatía } \\
\text { - } \text { Resiliención } \\
\text { - Auto reconocimiento } \\
\text { - Autoestima } \\
\text { - Constancia } \\
\text { - Ética y compromiso } \\
\text { con la realidad del } \\
\text { - } \text { Contexto } \\
\text { Proactividad }\end{array}$ & $\begin{array}{l}\text { - Saber técnicas de } \\
\text { marketing } \\
\text { - Administrar el recurso } \\
\text { humano, financiero, } \\
\text { físico } \\
\text { - Administrar el talento } \\
\text { humano } \\
\text { - Innovar } \\
\text { - Identificar nichos de } \\
\text { - mercado } \\
\text { Crear estrategias, } \\
\text { conocer el mercado }\end{array}$ & $\begin{array}{l}\text { - Habilidad para vender } \\
\text { - Trabajo bajo presión } \\
\text { - Manejo de la } \\
\text { incertidumbre } \\
\text { - Recepción a las } \\
\text { recomendaciones, } \\
\text { pedir ayuda } \\
\text { - Capital social fuerte } \\
\text { - Que se dé a reconocer } \\
\text { como una persona que } \\
\text { emprende } \\
\text { - Que convenza con su } \\
\text { idea (liderazgo) } \\
\text { - Capacidad de } \\
\text { coordinación }\end{array}$ & $\begin{array}{l}\text { - Trabajo en equipo } \\
\text { - Buenas relaciones } \\
\text { con el entorno } \\
\text { - Comunicación } \\
\text { activa }\end{array}$ \\
\hline
\end{tabular}

Fuente: elaboración propia a partir de los datos de fuentes primarias.

A continuación se destacan las recomendaciones de un funcionario de MinTIC para un emprendedor:

Debe estudiar mucho, importante que sepa de tendencias, que sepa para dónde va el mundo, que lea mucho, pero el mercado, a diferencia del 2000 o del 2002, ya está muy saturado, entonces hay que realmente ser innovador, pensar fuera de la caja, implementar tecnologías que no se han usado, resolver problemas de maneras que no se han resuelto, para así sacarle provecho a la tecnología.

La tecnología es muy importante, pero no es el fin, es el medio, no piensen en la App, piensen que la App les va a solucionar un problema. Lo más importante son los problemas que vamos a solucionar, después miran cómo, pero lo más importante es el problema que se va a resolver.

Que se equivoque y que se equivoque mucho, el valor del fracaso es muy importante en el emprendimiento, hay compañías, hay muchas fuentes que no invierten en compañías que no se hayan quebrado o que no hayan fracasado, el valor del fracaso es muy importante. Tengan en cuenta que equivocarse no está mal, tenemos una cultura de renuencia al fracaso, de verlo como algo malo y simplemente es la oportunidad de que aprendan, que aprendan muchísimo más de lo que aprenderían siendo exitosos, entonces no tengan miedo a equivocarse, prototipen, validen, testeen, apóyense en las entidades que tienen, es mucho más fácil que hacerlo solo, aprovechen. (transcripción entrevista, 2018)

\section{Tendencias de emprendimiento, tecnología e innovación}

Las siguientes temáticas se valoraron de acuerdo con la importancia que tiene para los actores en el presente y futuro para el desarrollo de la región. La escala va de 1 a 5, siendo 5 la calificación más alta: 


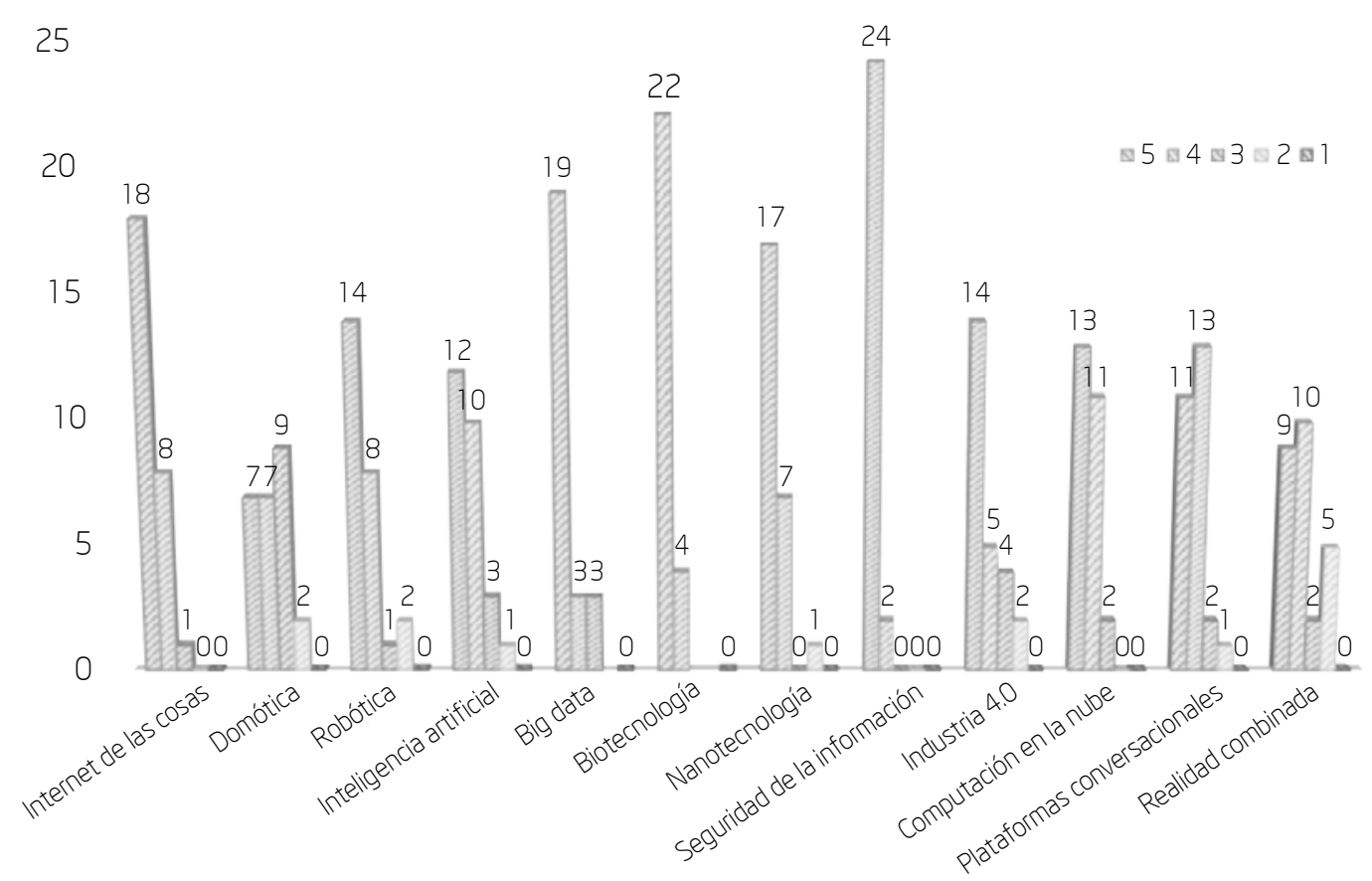

Figura 2. Tendencias de emprendimiento, tecnología e innovación cuantificado según actores externos

Fuente: elaboración propia a partir de los datos de fuentes primarias.

\section{Como lo muestra la figura 2, la seguridad de la Alianzas estratégicas en torno información es la temática que más valoración obtuvo al emprendimiento} en las entrevistas, seguida por la biotecnología, big data e IoT (internet de las cosas). En cuanto a los A continuación, la figura 3 muestra los países temas menos valorados por los entrevistados está la que los actores enunciaron con los cuales tienen domótica y la realidad combinada. alianzas estratégicas en torno al emprendimiento y la innovación:

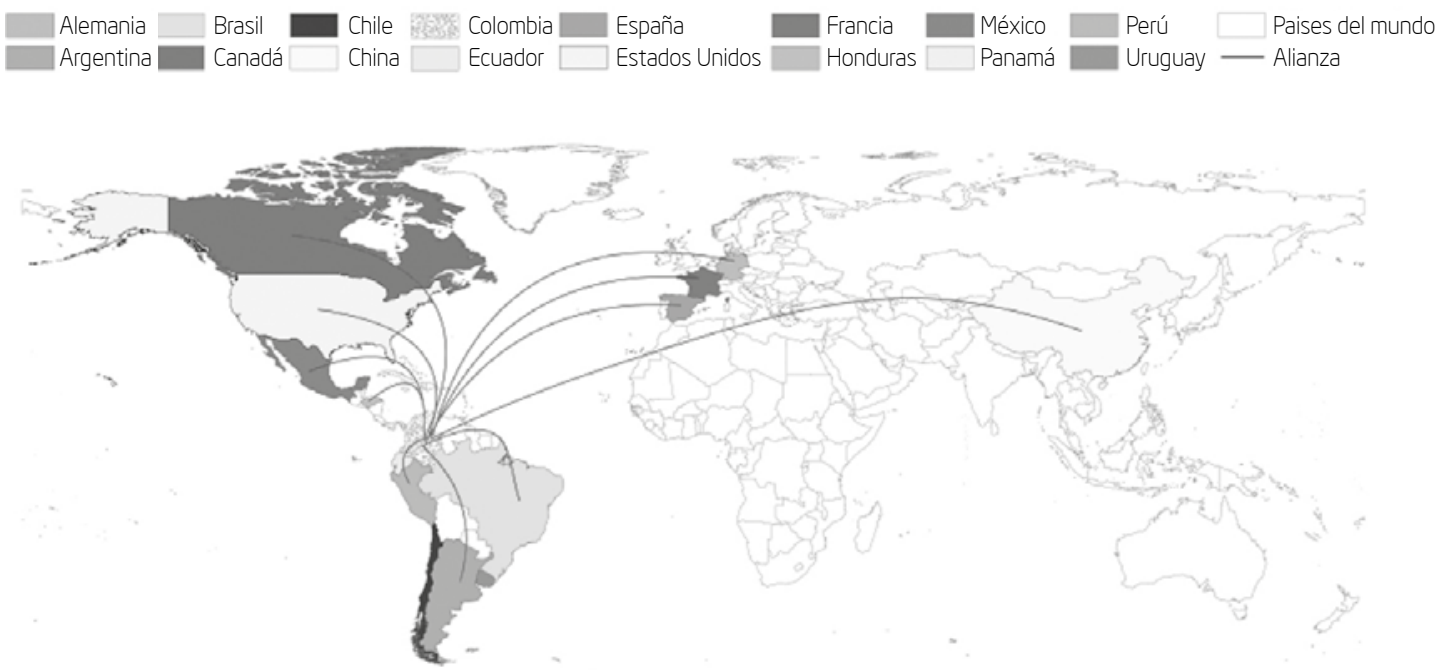

Figura 3. Alianzas estratégicas internacionales en torno al emprendiendo de Colombia

Fuente: elaboración propia 


\section{Conclusiones}

El acercamiento con los actores externos dejó ver el gran interés que existe en materia de innovación y emprendimiento y la necesidad que tiene el sector empresarial de alcanzar una mejor articulación con la academia para resolver problemáticas e innovar en pro de su sostenibilidad y competitividad. Esto permitió definir como elementos fundamentales para el modelo, en cuestión de articulación con el entorno: vigilancia tecnológica y demanda de los interesados.

En este mismo sentido, fue relevante reconocer que los intereses se enfocan en formación y mentoría, lo cual permitió definir como elemento central del modelo la articulación entre el Proyecto Educativo Institucional (PEI), la investigación, el desarrollo tecnológico y la apropiación social del conocimiento. Se propuso como fundamento para dicha articulación la gestión del conocimiento.

En relación con el desarrollo de competencias y habilidades, se identificó que es necesario formar seres humanos capaces de cooperar en lugar de competir, de resolver problemáticas complejas, de asimilar el cambio permanente, de afrontar y aprovechar las oportunidades del cambio tecnológico, pero sobretodo, seres con ética y valores que piensen siempre en las generaciones futuras. Con respecto al rol de las Instituciones de Educación Superior (IES), fue determinante descubrir que sus funciones misionales se deben desarrollar no mediante un rol pasivo, sino en articulación con todos los actores, para así asimilar y buscar soluciones a las problemáticas de la sociedad. En este sentido, el EBT es apenas una de las múltiples oportunidades que se pueden entrever en la ruta que marcan las nuevas revoluciones tecnológicas.

La revisión conceptual también permitió identificar que afrontamos hoy un cambio estructural que atraviesa nuestra sociedad, en este destacan conceptos como la economía digital, la economía circular, el blockchain, la inteligencia artificial y otros derivados de la convergencia tecnológica en las últimas décadas. Más allá de la preocupación por el futuro, hay un sinnúmero de posibilidades que brinda la tecnología y que permiten prever con mayor exactitud fenómenos de toda naturaleza y que marcan nociones sobre cómo cambiar el futuro.

Finalmente, se reconoció que el verdadero reto es contar con esquemas de gobernanza claros en los ecosistemas de $\mathrm{I}+\mathrm{D}+\mathrm{i}$, con instituciones sólidas e individuos capaces de afrontar, no solo el cambio tecnológico, sino el humano. Ante las nuevas revoluciones industriales se facilita la transferencia tecnológica, pues si bien, el mundo está interconectado, cada territorio tiene sus particularidades y necesidades específicas que pueden generar sinergia y cooperación con otros.

\section{Referencias}

Aboal, D., Álvarez, R., y Grazzi, M. (2019, marzo 18). ¿Qué sabemos y qué nos gustaría saber sobre la economía de la innovación y el emprendimiento? BID Mejorando vidas [entrada blog]. Recuperado de https://blogs.iadb.org/ innovacion/es/nuevas-investigaciones-en-laeconomia-de-la-innovacion/

Bustelo, M., y Raygada, G. (2019, febrero 21). ¿Qué rol jugarán las mujeres camino al 2050? BID Mejorando vidas [entrada blog]. Recuperado de https://blogs.iadb.org/igualdad/es/mujerescamino-al-2050/

Cámara de Comercio de Cali (ccc) (2018, diciembre). Ranking: Las 100+ de las iniciativas Cluster. Revista Acción, 210. Recuperado de https:// www.ccc.org.co/categoria_articulo/ranking-las100-las-iniciativas-cluster/

Carayannis, G.E., Barth, T. D., y Campbell, .D (2012). The Quintuple Helix innovation model: Global warming as a challenge and driver for innovation. Journal of Innovation and Entrepreneurship 1(2). https://doi. org/10.1186/2192-5372-1-2 
Departamento Nacional de Planeación (DNP) (2019). Plan de Desarrollo 2018-2022. Pacto por Colombia, pacto por la equidad. Recuperado de https://colaboracion.dnp.gov.co/CDT/Prensa/ Resumen-PND2018-2022-final.pdf

Díaz, E., Gómez, C., Cuervo, J., y Sota, J. (2007). Guía para emprendedores de base tecnológica. Emprender desde la investigación y el desarrollo tecnológico. Madrid: Elecé Industria Gráfica. Recuperado de file:///Users/meliremo/ Downloads/31_nebts.pdf

Domínguez, M., y García, F. (2009, julio-diciembre) La sexta revolución tecnológica: El camino hacia la singularidad en el siglo XXI. El Hombre y la Máquina, (33), 8-21. Recuperado de https:// www.redalyc.org/pdf/478/47812225002.pdf

Feldman y Kogler. (2008). The competitive advantage of regions and nations: Technology transfer through foreing direct investment. Great Britain: MPG Books Group.

Guerrero, M. (2018). La quinta revolución industrial. Kaizen, Mejora Continua Industria 4.0, Excelencia Operativa, Estrategias Lean, Kaizen y mucho más [entrada blog]. Recuperado de https://manuelguerrerocano.com/quintarevolucion-industrial-singularidad/

Guerrero, M., y Urbano, D. (2017). Emprendimiento e innovación: realidades y retos de las universidades españolas. Economía Industrial, (404), 21-30. Recuperado de https://dialnet. unirioja.es/servlet/articulo?codigo $=6117389$

Guijosa, C. (2018, mayo 7). La innovación debe nacer en las universidades de investigación, según encuesta. Observatorio de Investigación Educativa. Recuperado de https://observatorio. itesm.mx/edu-news/innovacion-universidadesde-investigacion?format $=$ amp\&_twitter_ impression=true

Huete, J. (2018, enero, 17). MIDE quiere situar a Madrid en el pódium de la innovación europea. Innovaspain. Recuperado de https://www. innovaspain.com/mide-plataforma-madridinnovacion/
Ministerio de Educación y Ciencia (MEc) (2008) La creación de empresas de base tecnológica en el ámbito universitario a partir de la reforma de la LOU. Madrid: MEC.

Moncayo, E. (2002) Glocalización: nuevos enfoques teóricos sobre el desarrollo regional (sub nacional) en el contexto de la integración económica y de la globalización. Desafios (7), 50-99.

Robotsia (2018) Singularidad tecnológica, ¿el fin de la humanidad?. History [en línea]. Recuperado de https://mx.tuhistory.com/noticias/singularidadtecnologica-el-fin-de-la-humanidad

Rodríguez, Z. H. (2007, junio) El paradigma de las competencias hacia la educación superior. Revista Facultad de Ciencias Económicas: Investigación y Reflexión, XV(1), 45-165.

Rubio de las Alas-Pumariño, T. (2014). Recomendaciones para mejorar el modelo de transferencia de tecnología en las universidades españolas. Conferencia de Consejos Sociales. Colección estudios e informes 05. Las Palmas de Gran Canaria: Advantia Comunicación Gráfica. Recuperado de http://www.oepm.es/export/ sites/oepm/comun/documentos_relacionados/ Publicaciones/monografias/2014_12_01_ Recomendac_mejora_modelo_de_transferencia_ Univ.pdf

Segarra Ciprés, M., Grangel Seguer, R., Belmonte Fernández, O., y Aguado González, S. (2017, enero-abril). ¿Cómo potenciar el emprendimiento y la innovación mediante el desarrollo de proyectos de base tecnológica en el contexto docente universitario? CEF. Revista Tecnología, Ciencia y Educación, (6), 11-33. Recuperado de http://repositori.uji.es/ xmlui/bitstream/handle/10234/170893/52892. pdf? sequence $=4$

Zorro, C. (2015, julio-diciembre). Las perspectivas de la glocalización en un mundo cambiante: Una mirada desde Colombia. Revista internacional de cooperación y desarrollo, 2(2). 